\title{
Oral Bioavailability of Insulin Contained in Polysaccharide Nanoparticles
}

\author{
Bruno Sarmento, ${ }^{*}, \dagger$ António Ribeiro, ${ }^{\ddagger}$ Francisco Veiga, ${ }^{\ddagger}$ Domingos Ferreira, ${ }^{\dagger}$ and \\ Ronald Neufeld§ \\ Department of Pharmaceutical Technology, Faculty of Pharmacy of the University of Porto, \\ 4050-047 Porto, Portugal, Department of Pharmaceutical Technology, Faculty of Pharmacy of the \\ University of Coimbra, Coimbra, Portugal, and Chemical Engineering Department, Queen's University, \\ Kingston, Ontario, Canada K7L 3N6
}

Received April 7, 2007; Revised Manuscript Received July 21, 2007

\begin{abstract}
The pharmacological activity of insulin-loaded dextran sulfate/chitosan nanoparticles was evaluated following oral dosage in diabetic rats. Nanoparticles were mucoadhesive and negatively charged with a mean size of 500 $\mathrm{nm}$, suitable for uptake within the gastrointestinal tract. Insulin association efficiency was over $70 \%$ and was released in a $\mathrm{pH}$-dependent manner under simulated gastrointestinal conditions. Orally delivered nanoparticles lowered basal serum glucose levels in diabetic rats around 35\% with 50 and $100 \mathrm{IU} / \mathrm{kg}$ doses sustaining hypoglycemia over $24 \mathrm{~h}$. Pharmacological availability was 5.6 and $3.4 \%$ for the 50 and $100 \mathrm{IU} / \mathrm{kg}$ doses, respectively, a significant increase over $1.6 \%$, determined for oral insulin alone in solution. Confocal microscopic examinations of FITC-labeled insulin nanoparticles showed adhesion to rat intestinal epithelium, and internalization of insulin within the intestinal mucosa. Encapsulation of insulin into dextran sulfate/chitosan nanoparticles was a key factor in the improvement of the bioavailability of its oral delivery over insulin solution.
\end{abstract}

\section{Introduction}

Among the controlled release formulations, polymeric colloidal systems have shown a certain degree of success for the oral delivery of therapeutic proteins like insulin, ${ }^{1,2}$ calcitonin, ${ }^{3}$ octeotride, ${ }^{4}$ and cyclosporine, 5 among others. Nanoparticles formulated from natural polymers like alginate, agar, agarose, chitosan, or synthetic polymers such as poly(lactic acid), poly(lactic-co-glycolic acid), poly( $\epsilon$-caprolactone), and poly(phosphoesters) have attracted significant interest as protein carriers. ${ }^{6-8}$

The gastrointestinal (GI) uptake of proteins like insulin can be improved by association to nanoparticles, mainly to protect insulin from degradation in the GI tract. Low $\mathrm{pH}$ and protease hydrolysis have demonstrated to be an important limitation to intestinal absorption of intact insulin ${ }^{9}$ and transport into systemic circulation. ${ }^{6}$ These carriers have been improving oral peptide delivery due to their prolonged retention in the GI tract and excellent penetration into the mucus layer. ${ }^{10}$ Further, they are able to be taken up by the M cells of the Peyer's patches, a type of lymphatic island within the intestinal tract that represents the major gateway through which nanoparticles may be absorbed. ${ }^{11}$ However, the mechanisms by which nanoparticles based on natural polysaccharides have improved the oral absorption of proteins still needs further elucidation. ${ }^{12}$

Polysaccharides are natural biodegradable hydrophilic polymers, which exhibit enzymatic degradation behavior and good biocompatibility. They can also form polyelectrolyte complexes by electrostatic interactions between oppositely charged groups resulting in ion-pairing without altering the integrity of the polymer. ${ }^{13}$ Nanoparticle delivery systems that have been

* Corresponding author. E-mail: bruno.sarmento@ff.up.pt. Tel: +351 222078949. Fax: +351 222073977.

University of Porto.

$\doteqdot$ University of Coimbra.

$\S$ Queen's University. developed by employing natural polysaccharides have the potential to retain protein stability, increase the duration of the therapeutic effect of proteins as well as the possibility of administration through nonparenteral routes. ${ }^{14}$ Such polymers can be easily modified chemically and biochemically, and are highly stable, safe, nontoxic, with gel forming properties, suggesting their suitability to be used for oral protein delivery. ${ }^{15}$ Chitosan (Chit), the most widely employed natural polysaccharide, is able to reduce the transepithelial electrical resistance, promoting the passage of small electrolytes and transiently opening tight conjunction between epithelial cells ${ }^{16}$ and to combine with anionic sialic acid residues of the intestinal mucosa ${ }^{17}$ due to mucoadhesive properties. The adhesion of Chit at the site of insulin GI absorption may offer various advantages for its uptake. ${ }^{18,19}$ Previous work with Chit coated alginate nanoparticles ${ }^{20}$ and other Chit-based nanoparticles ${ }^{2,21}$ demonstrated their potential to orally administer insulin, but those formulations also showed premature gastric insulin release, exposing insulin to undesired GI degradation.

Dextran sulfate (DS) is a biodegradable and biocompatible branched negatively charged polyanion able to strongly interact with positively charged proteins. Chit and DS have been complexed to formulate different drug delivery systems, ${ }^{22-25}$ but their application to oral delivery of proteins has not been explored. They are intrinsically hydrophilic, which may contribute to the encapsulation of water-soluble proteins and to promote longer circulation times in vivo. Recently, we formulated insulin into DS/Chit nanoparticles, which fully retained insulin in simulated gastric medium, and sustained the release up to $24 \mathrm{~h}$ in simulated intestinal medium, while preserving its bioactivity. ${ }^{26}$

The main objective of the present study was to determine whether insulin encapsulated in DS/Chit nanoparticles induced a hypoglycemic effect after oral administration to diabetic rats. The use of a single nanoparticle administration is the most 

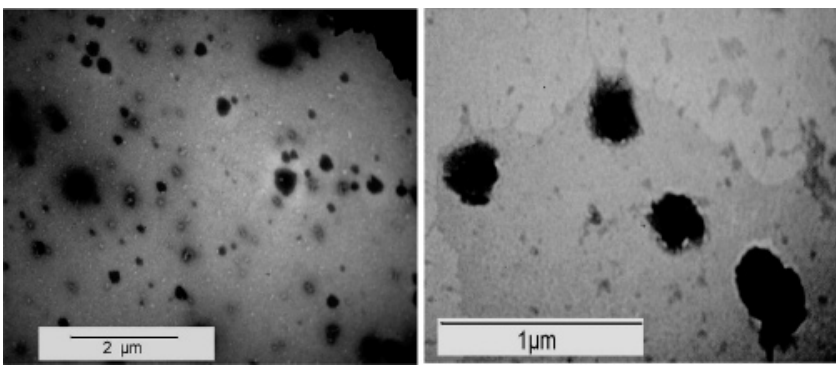

Figure 1. TEM image of DS/Chit nanoparticles (DS:Chit mass ratio of $1.5: 1)$.

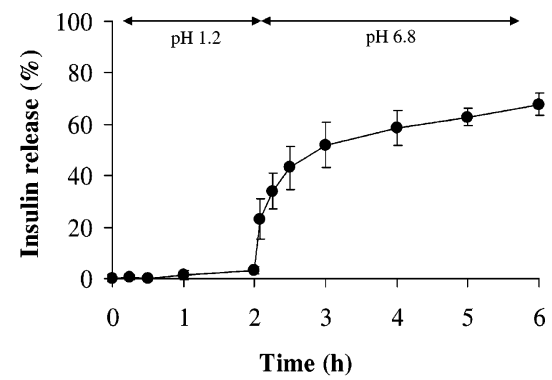

Figure 2. Insulin release from insulin DS/Chit nanoparticles produced with a DS:Chit mass ratio of $1.5: 1$ in gastric $\mathrm{pH} 1.2$ simulated fluid for $2 \mathrm{~h}$ followed by additional $4 \mathrm{~h}$ in intestinal $\mathrm{pH} 6.8$ simulated fluids at $37^{\circ} \mathrm{C}$ (mean $\pm \mathrm{SD}, n=3$ ).

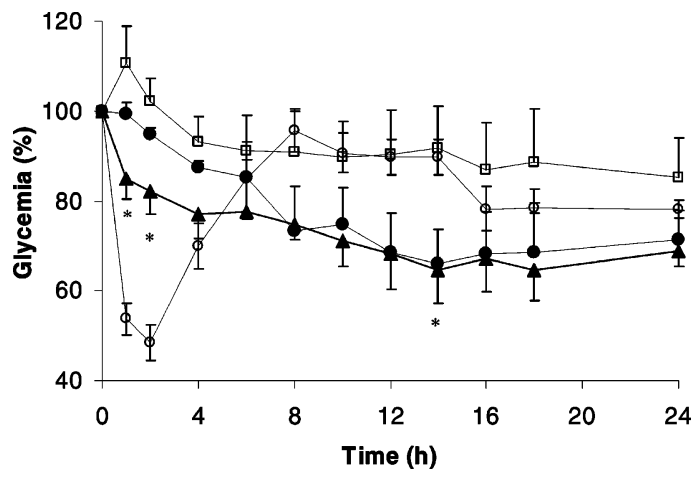

Figure 3. Plasma glucose levels after administration of insulin-loaded nanoparticles produced with a DS:Chit mass ratio of 1.5:1 insulinloaded $50(\bullet)$ and $100(\Delta) \mathrm{IU} / \mathrm{kg}$ and empty nanoparticles ( $\square$ ), compared to $2.5 \mathrm{IU} / \mathrm{kg}$ delivered subcutaneously $(O)$. Data represent the mean \pm SEM, $n=6$ per group. ${ }^{*} 100 \mathrm{IU} / \mathrm{kg}$ dose showed statistically significant differences from negative empty nanoparticle control $(p<0.05)$.

common way of evaluating the oral insulin bioavailability, mostly because this assay is performed in fasted animals. Prolonged assay times could lead to false decrease of blood glucose levels due to absence of food. ${ }^{27}$ Moreover, it was demonstrated that blood glucose levels of diabetic rats can be effectively controlled by insulin-loaded microparticle administration, following either single or multiple oral administration. ${ }^{28}$ Also, to predict the mechanism of insulin absorption from the GI tract, fluorescent-labeled insulin associated with nanoparticles was examined.

\section{Experimental Section}

Materials. Low MW Chit $(\approx 50 \mathrm{kDa})$, degree of acetylation $15 \%$, was purchased from Sigma. High MW DS (500 kDa) was obtained from PKC. DS stock solutions were prepared in deionized water (MilliQ) overnight under magnetic stirring and Chit was dissolved in $1 \%$ acetic acid solution in deionized water followed by filtering using a Millipore \#2 paper filter and stored at $4{ }^{\circ} \mathrm{C}$. Human crystalline zincinsulin (Lot RS0325, $7.0 \mathrm{mg}$ lyophilized human biosynthetic insulin per vial) was a gift from Lilly Portugal. Wistar male rats weighing 200-250 g, were provided by Charles River, Barcelona, Spain. Streptozocin and Insulin-FITC from bovine pancreas were from Fluka. Alexa Fluor 594 was from Molecular Probes. Human insulin detecting ELISA kits were from Linco.

\section{Methods}

Preparation of Nanoparticles. Optimized conditions to obtain DS/ Chit nanoparticles were established previously. ${ }^{26}$ Briefly, nanoparticle complexation between DS and Chit was performed by employing aqueous solutions of oppositely charged polymers in a final volume of $120 \mathrm{~mL}$. Unless otherwise mentioned, complexes were obtained after dropwise addition of Chit solution at $\mathrm{pH} 5.0$ to DS solution at $\mathrm{pH} 3.2$ under magnetic stirring followed by additional mixing for $15 \mathrm{~min}$ at $600 \mathrm{rpm}$ to final concentrations of $0.15 \%$ DS and $0.10 \%$ Chit (DS: Chit mass ratio 1.5:1). For insulin encapsulation, the protein was previously dissolved in DS solution before Chit complexation.

Nanoparticle Characterization. Particle size was measured by photon correlation spectroscopy (PCS) at $25^{\circ} \mathrm{C}$ with a detection angle of $90^{\circ}$ and $\zeta$ potential by laser doppler anemometry (LDA) using a Malvern Zetasizer and Particle Analyzer 5000 (Malvern Instruments) $(n \geq 6)$.

Morphology was evaluated by placing samples in a grid, treating with uranil acetate and observing under a Zeiss TEM (model 902A)

Association efficiency (AE) as the amount of insulin associated with the particles was calculated by the difference between the total amount used to prepare the particles, and the amount of residual insulin in the supernatant after particle centrifugation. The difference between the total initial insulin and the amount of unassociated residual after particle separation, as the percentage of total nanoparticle dry mass is referred to as loading capacity (LC) (=3).

Insulin Release from Nanoparticles. Insulin-loaded nanoparticles (200 mg) were placed into $20 \mathrm{~mL}$ of $\mathrm{HCl}$ buffer at $\mathrm{pH} 1.2$ (USP XXVIII) and incubated at $37^{\circ} \mathrm{C}$ for $2 \mathrm{~h}$ under magnetic stirring at 100 rpm. At determined times, $0.4 \mathrm{~mL}$ samples were taken for insulin determination and replaced by fresh medium. After $2 \mathrm{~h}$, the same nanoparticles were isolated by centrifugation $(20000 \mathrm{~g} / 15 \mathrm{~min})$ and were transferred to $20 \mathrm{~mL}$ of phosphate buffer at $\mathrm{pH} 6.8$ (USP XXVIII) and incubated at $37^{\circ} \mathrm{C}$ for an additional $4 \mathrm{~h}$ under magnetic stirring at 100 rpm. At determined times, $0.4 \mathrm{~mL}$ samples were taken for insulin determination and replaced by fresh medium. Release assays were performed in triplicate.

Insulin Determination. Insulin concentration was determined spectrophotometrically using the Coomassie PlusTM (Pierce, Rockford, MD) modified Bradford assay. ${ }^{29}$ Briefly, insulin samples and Bradford reagent were mixed at $1: 1 \mathrm{v} / \mathrm{v}$ ratio in a 96-well plate and incubated at room temperature for $15 \mathrm{~min}$. The absorbance was measured at 595 $\mathrm{nm}$ on a thermomax plate reader (PowerWaveX; Bio-Tek, Winooski, VT). Calibration curves were made using supernatant of unloaded

Table 1. Characterization of DS/Chit Nanoparticles Produced with Two Different DS:Chit Mass Ratios (1.5:1 and 1:1.5) in Terms of Mean Nanoparticle Size, $\zeta$ Potential, and Insulin AE and LC $(n=3$, Mean \pm SD)

\begin{tabular}{ccccc}
\hline & size $(\mathrm{nm})$ & $\xi$ potential $(\mathrm{mV})$ & AE $(\%)$ & LC $(\%)$ \\
\hline insulin-loaded nanoparticles $(1.5: 1)$ & $527 \pm 103$ & $-20.6 \pm 4.8$ & $69.3 \pm 1.3$ & $2.3 \pm 0.6$ \\
insulin-loaded nanoparticles $(1: 1.5)$ & $1577 \pm 230^{a}$ & $11.5 \pm 7.5$ & $24.0 \pm 1.7^{a}$ & $2.0 \pm 1.5$ \\
\hline
\end{tabular}

a Statistically significant between groups $(p<0.05)$. 
Table 2. Pharmacodynamic Parameters after Oral Administration of Insulin-Loaded Nanoparticles in Both 50 and 100 IU/kg Doses and Insulin Solution $50 \mathrm{IU} / \mathrm{kg}^{\mathrm{a}}$

\begin{tabular}{lcccc}
\hline & hypoglycemic effect $(\%)$ & $\mathrm{PA}(\%)$ & $\mathrm{T}_{\max }(\mathrm{h})$ & $\mathrm{C}_{\min }(\%$ basal glucose) \\
\hline insulin-loaded nanoparticles $(50 \mathrm{lU} / \mathrm{kg})$ & $22.0 \pm 10.5$ & $5.6 \pm 2.6^{b}$ & 14 & $66.8 \pm 10.6$ \\
insulin-loaded nanoparticles $(100 \mathrm{lU} / \mathrm{kg})$ & $27.3 \pm 3.8$ & $3.4 \pm 0.9$ & 14 & $64.5 \pm 7.3$ \\
oral solution $(50 \mathrm{lU} / \mathrm{kg})$ & $10.8 \pm 1.6$ & $1.6 \pm 0.7$ & 6 & $80.8 \pm 8.3$ \\
\hline
\end{tabular}

a Data represent the mean $\pm \mathrm{SD}, n=6$ per group. ${ }^{b}$ Statistically significant differences from oral insulin solution control $(p<0.05)$. Hypoglycemic effect determined on the basis of AAC. $T_{\max }$ : time at minimum relative basal glucose concentration in the blood, $C_{\text {min: }}$ minimum relative basal glucose concentration in the blood.

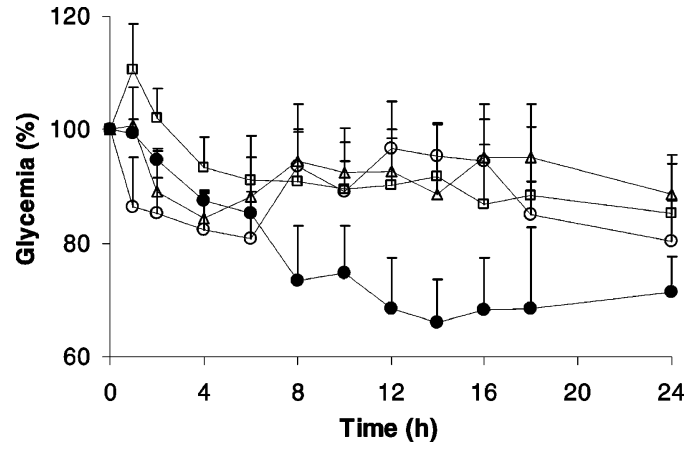

Figure 4. Plasma glucose levels after oral administration of insulinloaded nanoparticles produced with a DS:Chit mass ratio of 1.5:1 50 $\mathrm{IU} / \mathrm{kg}(\bullet)$, oral insulin solution $50 \mathrm{IU} / \mathrm{kg}(\bigcirc)$, empty nanoparticles $(\square)$, and suspension of empty nanoparticles in insulin solution $50 \mathrm{IU} / \mathrm{kg}$ $(\triangle)$. Data represent the mean \pm SEM, $n=6$ per group.

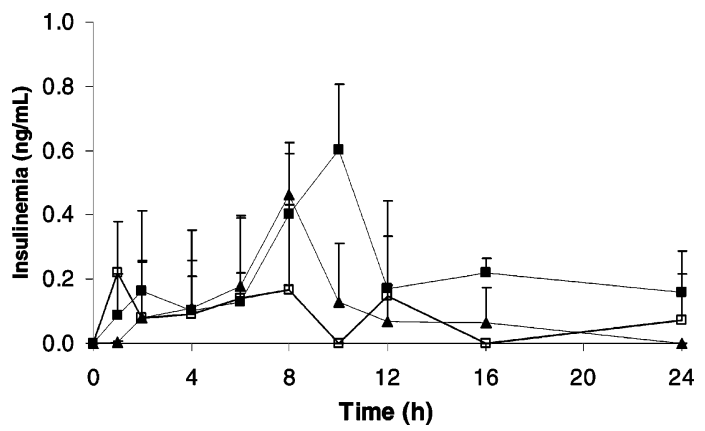

Figure 5. Serum insulin levels after oral administration of insulinloaded nanoparticles produced with DS:Chit mass ratio of 1.5:150

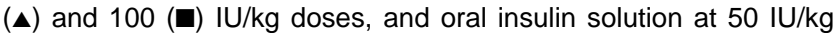
( $\square$ ). Data represent the mean $\pm \mathrm{SD}, n=6$ per group.

particles for $\mathrm{AE}$ and unloaded nanoparticles for in vitro release profile. All the assays were performed in triplicate.

Insulin Pharmacological Activity. Insulin pharmacological activity was determined in diabetic rats following the protocol described previously. ${ }^{30}$ Nanoparticles with DS:Chit mass ratios of 1.5:1 and 1:1.5 were administered at insulin doses of 50 or $100 \mathrm{IU} / \mathrm{kg}$. Control rats were administered an equivalent volume of insulin solution (50 IU/ $\mathrm{kg}$ ), or a suspension of empty nanoparticles in insulin solution (50 IU/ $\mathrm{kg}$ ), or a dispersion of blank nanoparticles. The volume of dispersion and controls administered was $1.0 \mathrm{~mL}$ and formulations were diluted in $\mathrm{pH} 4.5$ phosphate buffer whenever required. Also, a control using subcutaneously injected insulin $(2.5 \mathrm{IU} / \mathrm{kg})$ was used. Aliquots were collected before and during $24 \mathrm{~h}$ period following administration.

Plasma glucose levels were plotted against time to evaluate the cumulative hypoglycemic effect over time after insulin administration, quantified by the area above the curve (AAC), determined using the trapezoidal method. Pharmacological availability (PA) of peroral insulin loaded into nanoparticles and in solution was determined as the relative measure of the cumulative reduction in glucose blood levels compared to a $100 \%$ availability of the control insulin administered subcutaneously at a dose of $2.5 \mathrm{IU} / \mathrm{kg}$. Plasma insulin levels were plotted against time to evaluate the cumulative amount of insulin delivered to the plasma after insulin administration, quantified by area under the curve (AUC).
Plasma glucose level was determined using the Medisense Precision Xceed Kit (Abbot, range 10-600 mg/dL) and expressed as the percentage of the baseline plasma glucose level. The serum insulin concentration was measured by ELISA. Results are shown as the mean $( \pm \mathrm{SD})$ of at least six animals.

Tracking FITC-Labeled Nanoparticles. Fluorescein (FITC)-labeled insulin was loaded into nanoparticles to yield $2.0 \mathrm{mg}$ of FITC-insulin/ $\mathrm{mL}$ of nanoparticle suspension and administered by oral gavage $(150$ $\mathrm{mg}$ of nanoparticles diluted in $1.0 \mathrm{~mL}$ of $\mathrm{pH} 4.5$ phosphate buffer) to Wistar diabetic rats fasted overnight. The rats were sacrificed $3 \mathrm{~h}$ later and intestinal segments localized after laparotomy. After washing with isotonic saline, intestinal epithelial cell membranes were stained with Alexa Fluor 594 (0.05\% in PBS:glycerol 1:1) for $1 \mathrm{~h}$. Tissue was rinsed, mounted on glass slides and observed using a confocal laser scanning microscope (Leica). Tissue samples were scanned in the $x, y$ plane with a $z$-step of $500 \mathrm{~nm}$. Because the spectra are not overlapping, FITCinsulin and Alexa Fluor could be analyzed simultaneously with 488 and $561 \mathrm{~nm}$ laser, respectively. Control rats were dosed with FITCinsulin solution or empty nanoparticles.

Statistical Analysis. A one-way analysis of variance (ANOVA) was used to evaluate group comparison. If the group by each time interaction was significantly different $(p<0.05)$, differences between groups were compared within a post-hoc test $(\mathrm{S}-\mathrm{N}-\mathrm{K})$. All statistical analyses were performed with the SPSS software package (SPSS for Windows 14.0, SPSS, Chicago, IL).

\section{Results and Discussion}

DS/Chit colloidal nanoparticles were developed and optimized to encapsulate insulin for oral delivery. ${ }^{26}$ The mucoadhesive and absorption enhancement properties of Chit and the protective effect of DS against insulin release at low $\mathrm{pH}$ were combined to promote and ensure insulin intestinal absorption. Low MW and low degree of acetylation Chit was used because it was previously demonstrated that lower molar mass Chit leads to smaller particle size by polyelectrolyte complexation and low acetyl content favors the formation of less swollen particles. High MW DS was used as it resulted in more stable colloidal nanoparticles. ${ }^{13,31}$ Nanoparticles prepared with DS:Chit of 1.5:1 showed size around $500 \mathrm{~nm}$ (Table 1), resulting in a dense matrix with irregular spherical structure as seen in Figure 1. This irregular and highly aggregated structure is attributed to a strong interaction between polyions, ${ }^{31,32}$ occurring rapidly by random incorporation of different polymeric chains into the particle structure.

The $\zeta$ potential was less negative after encapsulation of insulin (-20.6 against $-24.5 \mathrm{mV}$ for insulin-free nanoparticles, Table 1 ), probably due to the localization of some insulin on the surface on the nanoparticles. At $\mathrm{pH} 5$ used for nanoparticle formulation, insulin is positively charged, affecting the surface charge of the nanoparticles, although to a small extent because of low loading. The AE found was $69.3 \%$ (Table 1), higher than that obtained with Chit nanoparticles without DS complexation. ${ }^{2,33}$ Particles with a DS:Chit mass ratio of $1: 1.5$ were also prepared, presenting higher particle size, around $1500 \mathrm{~nm}$, 
Table 3. Pharmacokinetic Parameters for Serum Insulin Levels after Oral Administration of Insulin-Loaded Nanoparticles in Both 50 and $100 \mathrm{IU} / \mathrm{kg}$ Doses and Insulin Solution $50 \mathrm{IU} / \mathrm{kg}^{\mathrm{a}}$

\begin{tabular}{lcrr}
\hline & bioavailability $[\mathrm{ng} /(\mathrm{mL} \mathrm{h})]$ & $T_{\max }(\mathrm{h})$ & $C_{\max }(\mathrm{ng} / \mathrm{mL})$ \\
\hline insulin-loaded DS/Chit nanoparticles $50 \mathrm{IU} / \mathrm{kg}$ & $2.46 \pm 1.47$ & 8 & $0.46 \pm 0.16$ \\
insulin-loaded DS/Chit nanoparticles $100 \mathrm{IU} / \mathrm{kg}$ & $5.25 \pm 2.14$ & 10 & $0.60 \pm 0.20$ \\
oral solution (50 IU/kg) & $1.75 \pm 0.87$ & 1 & $0.22 \pm 0.16$ \\
\hline
\end{tabular}

a Data represent the mean $\pm \mathrm{SD}, n=6$ per group. Bioavailability determined on the basis of AUC. $T_{\max }$ : time at maximum concentration of insulin in the blood. $C_{\text {max }}$ : maximum concentration of insulin in the blood.

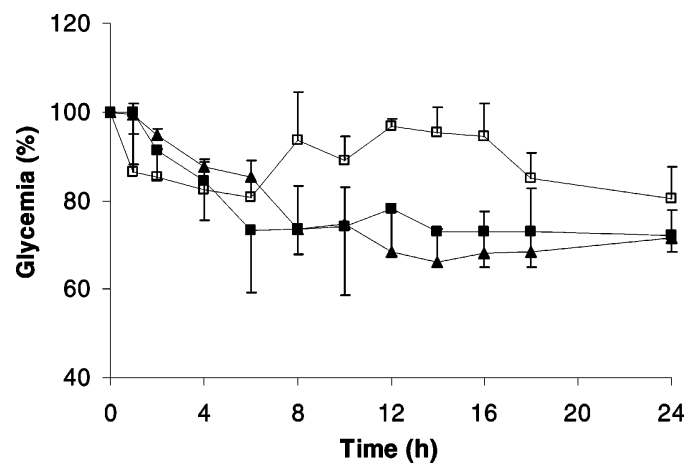

Figure 6. Plasma glucose levels after oral administration of different insulin-loaded nanoparticles prepared with DS:Chit mass ratio of 1:1.5

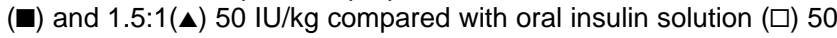
$\mathrm{IU} / \mathrm{kg}$. Data represent the mean $\pm \mathrm{SEM}, n=6$ per group.

positive charge, and lower insulin AE. Nevertheless, these nanoparticles were important for evaluating the potential for improved mucoadhesivity and the capacity of the higher Chit composition to transiently open tight junctions between epithelial cells, improving the absorption of insulin.

The retention of insulin in gastric fluids followed by intestinal media, simulating the passage from stomach to intestine was investigated. Figure 2 shows that insulin was retained at gastric $\mathrm{pH}$ for $2 \mathrm{~h}$. Following a $\mathrm{pH}$ change to 6.8, a two-step release pattern was observed, characterized by an initial rapid release during the first hour followed by a delayed release up to $6 \mathrm{~h}$. Release of insulin at gastric followed by neutral $\mathrm{pH}$ appears to occur by a slow dissociation of insulin from the Chit/DS polymers and is $\mathrm{pH}$-dependent. Ideally, insulin remains associated with nanoparticles in gastric conditions, and it is only released at the intestinal $\mathrm{pH}$ where the absorption potential is optimal. Compared to other Chit-based nanoparticle formulations, and especially alginate/chitosan nanoparticles, ${ }^{20,34}$ it may be deduced that DS prevented premature release of insulin by providing higher insulin retention capacity than alginate at gastric $\mathrm{pH}$, predicting lower insulin gastric degradation and higher availability of active insulin for absorption in the intestine.

The pharmacological effect of insulin loaded nanoparticles prepared with a DS:Chit mass ratio of $1.5: 1$ was evaluated in diabetic rats dosed orally with 50 and $100 \mathrm{IU} / \mathrm{kg}$, and changes in plasma glucose compared to those dosed subcutaneously with free insulin at $2.5 \mathrm{IU} / \mathrm{kg}$ and orally with empty nanoparticles. As observed in Figure 3, insulin nanoparticles were found to be effective in lowering the blood glucose levels at doses of 50 and $100 \mathrm{IU} / \mathrm{kg}$. The hypoglycemic effect appeared more pronounced after $6 \mathrm{~h}$ for a dose of $50 \mathrm{IU} / \mathrm{kg}$, but a faster onset was observed for a dose of $100 \mathrm{IU} / \mathrm{kg}$. The values of cumulative hypoglycemic effect found for both groups treated with different insulin doses showed sustained lowering of the blood glucose levels, observed $8 \mathrm{~h}$ after administration up to $24 \mathrm{~h}$. Both doses resulted in minimum blood glucose, around $70 \%$ of the initial value, after $14 \mathrm{~h}$. Previously, alginate/chitosan nanoparticles administered at same insulin dose level ${ }^{30}$ also reduced blood glucose levels to their minimum point after $14 \mathrm{~h}$, but to just over $60 \%$ of the initial value. Nevertheless, the hypoglycemic effect was higher after $24 \mathrm{~h}$ for the DS/Chit nanoparticles and the PA found for both formulations were comparable, as confirmed from Table 2, reflecting similar relative cumulative reduction in glucose blood levels. These results suggest that DS/Chit nanoparticles are able to provide insulin absorption on a continuous basis and for a longer time period than alginate/ chitosan nanoparticles.

To investigate whether nanoparticles facilitated intestinal absorption of free insulin, rats were treated orally with insulin alone, and with empty nanoparticles suspended in insulin solution $(50 \mathrm{IU} / \mathrm{kg})$. A slight decrease in glycemia after administration of oral insulin solution may be seen in Figure 4 for the first $6 \mathrm{~h}$ after administration, returning to basal levels. The presence of empty nanoparticles did not enhance the minor hypoglycemic effect of oral insulin solution, and therefore did not act as insulin absorption enhancers. Only the encapsulation of insulin into nanoparticles and the resulting protective effect highlighted the role of nanoparticulate delivery of insulin in producing a pharmacological response.

DS/Chit nanoparticles appear to stabilize and protect entrapped insulin from degradation in the gastrointestinal track as free insulin did not induce a glycemic response. After administration, nanoparticles likely reached the upper small intestine. Due to the mucoadhesive properties of chitosan, nanoparticles adhere to the intestinal walls, starting the promotion of insulin absorption. In addition, ongoing adhesion is likely promoted by the viscous lumen and fasted animal conditions. It is hypothesized that the initial absorption of insulin is related to the high local insulin concentration favoring diffusion through the intestinal wall. Direct uptake of insulin has been ascribed to binding to specific insulin receptors in intestinal enterocytes ${ }^{35-37}$ following passage to the interstitial space and then to the blood circulation. It was noted previously that the receptors located on the upper small intestine were saturated with insulin alginate/ chitosan nanoparticles administered at a dose of $50 \mathrm{IU} / \mathrm{kg},{ }^{30}$ and thus the higher insulin concentration gradient at a dose of 100 IU/kg probably was not efficient to promote additional insulin absorption at this level. Therefore, the increase of physiologic effect when the dose increased from 50 to $100 \mathrm{IU} / \mathrm{kg}$ in the first $6 \mathrm{~h}$ may be understood as faster intestinal insulin absorption due to the increment of number of nanoparticles that arrive at the posterior small intestine where they can be directly taken up by Peyer's patches. ${ }^{6,38}$ The direct uptake of nanoparticles is thought to occur to a lesser extent by enterocytes, ${ }^{6}$ and mainly in the Peyer's patches of the posterior intestine, possible mechanisms contributing to the total amount of absorbed insulin. Higher insulin blood levels detected initially for a dose of 100 $\mathrm{IU} / \mathrm{kg}$, as seen in Figure 5, confirm the absorption of insulin to a higher extent. Subsequent continuous absorption of insulin is probably due to ongoing sustained release of insulin during nanoparticle adhesion to the intestinal walls, and mainly due to passage of nanoparticles to the posterior small intestine, contributing to the plateau level of hypoglycemia. Despite mucoadhesive properties, nanoparticles probably slide along the 

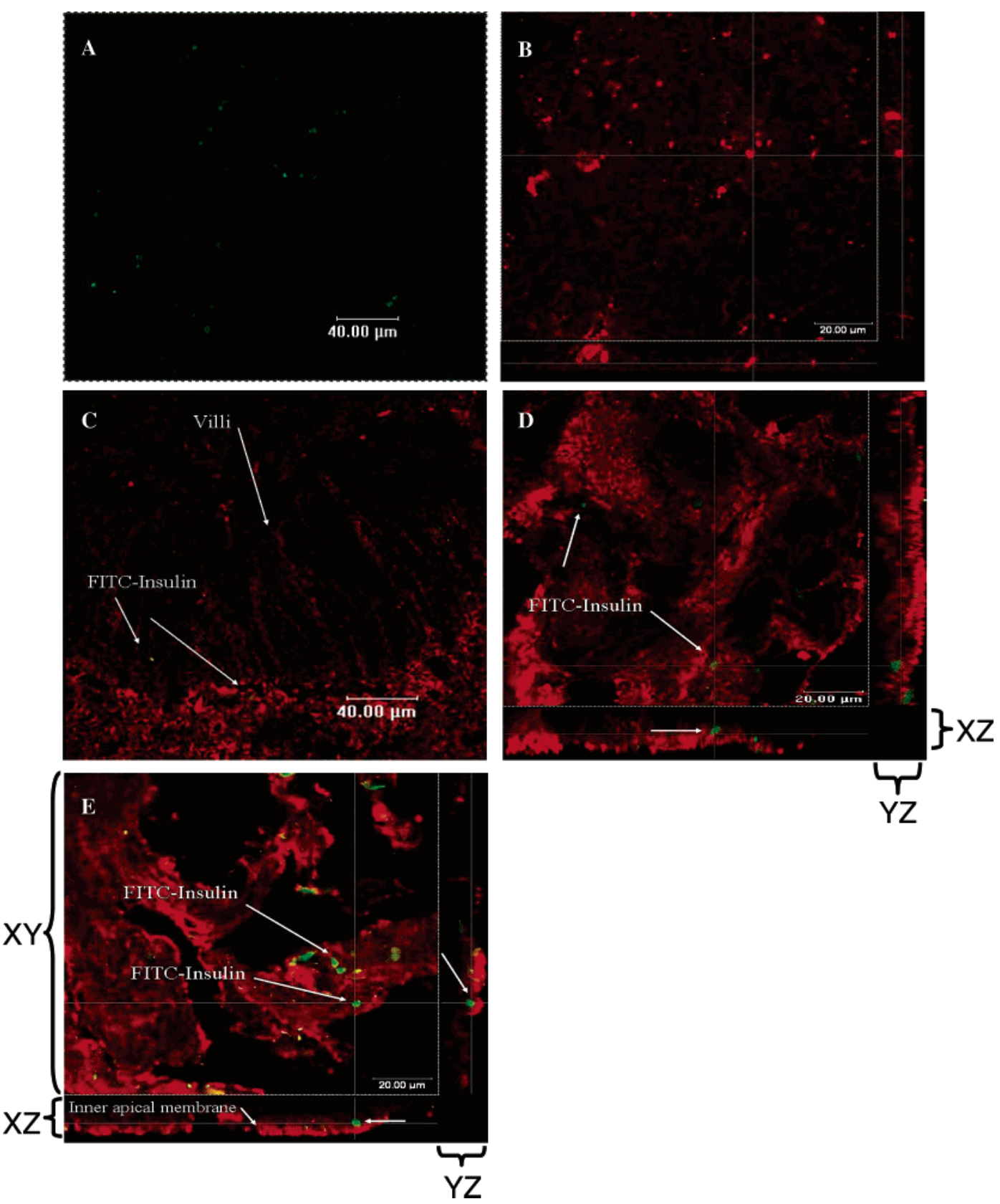

Figure 7. Confocal scanning microscopy images showing the association of FITC-insulin (green) to intestinal tissue (cell membranes in red). (A) FITC-insulin nanoparticle suspension. (B) Inner apical intestinal section treated with control empty nanoparticles. (C) Transversal section of intestinal epithelium. Longitudinal sections of anterior (D) and posterior (E) intestinal epithelium treated with insulin-loaded nanoparticles $(\sim 500 \mathrm{~nm})$. The intestinal line is demarked by the red line of cellular membranes. Also, green dots corresponding to the FITC-insulin (white arrows) can be observed both attached to the surface and inside the intestinal wall. The three-dimensional plan shows the internalization of labeled insulin. Gray lines indicate the plans where pictures were analyzed.

intestine maintaining their physical structure. In fact, Chit and Chit-based microspheres have shown mucoadhesive capacities in vitro, and concomitant slow upper small intestinal transit in vivo, but this intestinal retention was limited to a few hours ${ }^{39,40}$ and not affected by the particle size of the dosage form. ${ }^{39}$ The arrival of nanoparticles to the posterior intestine probably occurs in a few hours, and because adhesive properties are maintained, uptake is promoted.

Compared with the in vitro simulation, the extent of release in the gastrointestinal tract may be much less, and thus nanoparticles must be able to retain significant insulin loading when taken up. Uptake of the nanoparticles by the $\mathrm{M}$ cells on the Peyer's patches has been explained as responsible for longer hypoglycemic response. ${ }^{2,41}$ Furthermore, possible immunological consequences after oral insulin administration are believed to be absent. In fact, the prevalence of anti-insulin antibodies in diabetic people have been largely absent, mainly as a consequence of improvements in the purification of insulin. ${ }^{42}$

The hypoglycemic effect after $8 \mathrm{~h}$ was observed to be similar for both doses, different from that observed for the first hours of assay. It seems that the improvement of absorption was limited to the first hours when the entrance of nanoparticles to the posterior intestine was proportional to the number of nanoparticles administered. Nevertheless, the overall bioavailability of insulin, as outlined in Table 3, clearly demonstrated that it was higher when insulin was administered orally through nanoparticle delivery, in a dose of $100 \mathrm{IU} / \mathrm{kg}$, compared with a dose of $50 \mathrm{IU} / \mathrm{kg}$ and insulin solution at $50 \mathrm{IU} / \mathrm{kg}$, suggesting a relative dose/effect response. 
To evaluate the potential improvement of insulin absorption by nanoparticles composed by higher Chit composition, nanoparticles were prepared with a DS:Chit mass ratio of 1:1.5 and administered orally to diabetic rats. The results plotted in Figure 6 compare the cumulative hypoglycemic effect of the two different nanoparticle formulations prepared with DS:Chit mass ratio of 1.5:1 and 1:1.5. Formulation containing a higher fraction of Chit was able to decrease the blood glucose levels compared with the single insulin solution. However, only minor differences in the lowering of glucose levels and total cumulative hypoglycemic effect were found between nanoparticles prepared with DS:Chit mass ratio of 1.5:1 and 1:1.5. In the literature it is wellestablished that nanoparticle intestinal translocation increases when the particle diameter decreases. ${ }^{43}$ However, it is also reported that this trend is not absolute. Although lower than for nanoparticles with $100 \mathrm{~nm}$, the efficiency of nanoparticle uptake from rat intestinal tissue with Peyer's patches was very similar for nanoparticles of $500 \mathrm{~nm}$ and particles of 1 and 10 $\mu \mathrm{m} .{ }^{44}$ Also, the Caco- 2 cell uptake of nanoparticles was found very close for 500 and $1000 \mathrm{~nm}$ particles. ${ }^{45}$ The size range of nanoparticles is similar to that used in the present study and thus it is hypothesed that small differences would be expected between both formulations. In addition, theoretical improvement of insulin absorption would be obtained by nanoparticles prepared with a DS:Chit mass ratio of 1:1.5 due to improved mucoadhesivity because of the higher Chit fraction and reduced DS. It is possible that nanoparticles with a DS:Chit mass ratio of 1:1.5 stick strongly within the intestinal layer in the first hours after administration, resulting in a slightly lower blood glucose decrease compared to nanoparticles with a DS:Chit mass ratio of 1.5:1. However, nanoparticles prepared with a smaller faction of DS are probably less able to retain insulin in intestinal $\mathrm{pH}$ and protect it against degradation, ${ }^{26}$ suggesting once more the important role of DS on protection of insulin. Thus, it is possible that the protection of insulin within nanoparticles made of DS: Chit mass ratio 1:1.5 decreases compared with those of 1.5:1, resulting in less insulin absorption after $10 \mathrm{~h}$ through nanoparticle uptake.

Investigations on possible insulin absorption mechanisms were examined using FITC-insulin. Resulting FITC-insulin loaded nanoparticles with similar mean particle size of insulinloaded nanoparticles $(\sim 500 \mathrm{~nm})$ were administered orally to diabetic rats and intestinal epithelial sections observed through confocal microscopy and compared to rats dosed with empty nanoparticles.

As observed in Figure 7, FITC-insulin nanoparticles were located between villi (C) and on the surface and inside of anterior (D) and posterior intestinal epithelium (E). Intestinal rat section administered with empty nanoparticles (B) is shown as control where autofluorescence associated with the intestinal tissue was not observed.

The retention of insulin nanoparticles on the mucosa due to mucoadhesion and further insulin absorption appears to be demonstrated by the presence of FITC-insulin on the surface of villi (C) and on the apical membrane of the intestine (D, E) $3 \mathrm{~h}$ after administration. Further investigations revealed the presence of internalized FITC-insulin within the intestinal wall $(\mathrm{D}, \mathrm{E})$.

The anterior intestine region (D) appears to be less rich in FITC-insulin than the posterior region, which can indicate that insulin was internalized by different mechanisms depending on the depth that nanoparticles crossed the intestinal tract. Also, nanoparticles on the surface of intestinal mucosa were partially removed during the rinsing step before membrane staining, probably decreasing the observed fluorescence. In the anterior intestine, mucoadhesive nanoparticles probably attached to intestinal walls favoring the absorption of insulin, as hypothesized above. The mucoadhesive ${ }^{17}$ and absorption-enhancing properties of Chit ${ }^{16,46,47}$ may be fundamental to the insulin concentration on the surface of the intestinal wall and to its diffusion through the epithelium, promoting insulin absorption. Mucoadhesive nanoparticles penetrating the mucous layer are able to prolong the residence time and released insulin can interact deeply and permeate the intestinal barrier to the bloodstream.

The presence of green fluorescence in a great extent on the posterior intestine (E) seems to predict the presence of FITCinsulin in a deeper layer than the intestinal surface. The $\mathrm{XZ}$ and XY lateral planes clearly demonstrate that FITC-insulin is positioned below the apical membrane of the intestine, and therefore it was able to pass through the surface layer of enterocytes. Although it is not clear from Figure 7 that insulin nanoparticles were absorbed through Peyer's patches pathway, even though there is no specific fluorescent marker to identify $\mathrm{M}$ cells, ${ }^{48}$ there is evidence that the posterior ileum is the most prominent area of Peyer's patches, and the site where nanoparticles can be absorbed with higher efficacy. ${ }^{49,50}$

\section{Conclusion}

Insulin-loaded DS/Chit nanoparticles produced by polyelectrolyte complexation provided markedly enhanced intestinal absorption of insulin following oral administration. Nanoparticles lowered serum glucose levels of streptozotocin-induced diabetic rats at insulin doses of 50 and $100 \mathrm{IU} / \mathrm{kg}$ up to 67 and $64 \%$, respectively, of their basal glucose level. The hypoglycemic effect and insulinemia levels due to insulin nanoparticles were considerably higher than those obtained from oral insulin solution and suspension of empty nanoparticles in insulin solution, revealing 2-3-fold improvement of oral relative pharmacological availability. In addition, the hypoglycemic effect was observed for more than $24 \mathrm{~h}$. The mechanism of insulin absorption seems to be associated with insulin internalization, probably through vesicular structures in enterocytes and insulin-loaded nanoparticle uptake by Peyer patches, although no single mechanism could be identified. DS/Chit nanoparticles demonstrated promise as an oral delivery system for insulin and potentially for other therapeutic proteins.

Acknowledgment. This work was supported by Fundação para a Ciência e Tecnologia, Portugal and by the Natural Sciences and Engineering Research Council of Canada. We thank Paula Sampaio of IBMC for confocal microscopy experiments and Lilly Portugal for insulin supply.

\section{References and Notes}

(1) Qian, F.; Cui, F.; Ding, J.; Tang, C.; Yin, C. Chitosan graft copolymer nanoparticles for oral protein drug delivery: preparation and characterization. Biomacromolecules 2006, 7, 2722-2727.

(2) Ma, Z.; Lim, T. M.; Lim, L.-Y. Pharmacological activity of peroral chitosan-insulin nanoparticles in diabetic rats. Int. J. Pharm. 2005, 293, 271-280.

(3) Prego, C.; Torres, D.; Alonso, M. J. Chitosan Nanocapsules as Carriers for Oral Peptide Delivery: Effect of Chitosan Molecular Weight and Type of Salt on the In Vitro Behaviour and In Vivo Effectiveness. J. Nanosci. Nanotechnol. 2006, 6, 1-8.

(4) Thanou, M.; Verhoef, J. C.; Marbach, P.; Junginger, H. E. Intestinal absorption of octreotide: N-trimethyl chitosan chloride (TMC) ameliorates the permeability and absorption properties of the somatostatin analogue in vitro and in vivo. J. Pharm. Sci. 2000, 89, 951957. 
(5) El-Shabouri, M. H. Positively charged nanoparticles for improving the oral bioavailability of cyclosporin-A. Int. J. Pharm. 2002, 249, $101-108$.

(6) des Rieux, A.; Fievez, V.; Garinot, M.; Schneider, Y.-J.; Preat, V. Nanoparticles as potential oral delivery systems of proteins and vaccines: A mechanistic approach. J Control. Release 2006, 116, $1-27$.

(7) Soppimath, K. S.; Aminabhavi, T. M.; Kulkarni, A. R.; Rudzinski, W. E. Biodegradable polymeric nanoparticles as drug delivery devices. J. Control. Release 2001, 70, 1-20.

(8) Panyam, J.; Labhasetwar, V. Biodegradable nanoparticles for drug and gene delivery to cells and tissue. Adv. Drug. Deliv. Rev. 2003, $55,329-347$

(9) Aoki, Y.; Morishita, M.; Takayama, K. Role of the mucous/ glycocalyx layers in insulin permeation across the rat ileal membrane. Int. J. Pharm. 2005, 297, 98-109.

(10) Takeuchi, H.; Yamamoto, H.; Kawashima, Y. Mucoadhesive nanoparticulate systems for peptide drug delivery. Adv. Drug. Deliv. Rev. 2001, 47, 39-54

(11) Fasano, A. Innovative strategies for the oral delivery of drugs and peptides. Trends Biotechnol. 1998, 16, 152-157.

(12) Morishita, M.; Peppas, N. A. Is the oral route possible for peptide and protein drug delivery? Drug. Discov. Today 2006, 11, 905910.

(13) Schatz, C.; Domard, A.; Viton, C.; Pichot, C.; Delair, T. Versatile and efficient formation of colloids of biopolymer-based polyelectrolyte complexes. Biomacromolecules 2004, 5, 1882-1892.

(14) Florence, A. The oral absorption of Micro- and Nanoparticulates: Neither exceptional nor Unusual. Pharm. Res. 1997, 14, 259-266.

(15) Sinha, V. R.; Kumria, R. Polysaccharides in colon-specific drug delivery. Int. J. Pharm. 2001, 224, 19-38.

(16) Artursson, P. Effect of Chitosan on the Permeability of monolayers of Intestinal Epithelial cells (Caco-2). Pharm. Res. 1994, 11, 13581361.

(17) Lehr, C.-M.; Bouwstra, J. A.; Schacht, E. H.; Junginger, H. E. In vitro evaluation of mucoadhesive properties of chitosan and some other natural polymers. Int. J. Pharm. 1992, 78, 43-48.

(18) Thanou, M.; Verhoef, J. C.; Junginger, H. E. Chitosan and its derivatives as intestinal absorption enhancers. Adv. Drug. Deliv. Rev 2001, 50, 91-101.

(19) Thanou, M.; Verhoef, J. C.; Junginger, H. E. Oral drug absorption enhancement by chitosan and its derivatives. Adv. Drug. Deliv. Rev. 2001, 52, 117-126.

(20) Sarmento, B.; Ribeiro, A.; Veiga, F.; Neufeld, R.; Ferreira, D. In New alginate/chitosan nanoparticles for oral delivery of insulin, VII Spanish-Portuguese Conference on Controlled Drug Delivery Controlled Release Society, Pamplona, Spain, 2006.

(21) Pan, Y.; Li, Y. J.; Zhao, H. Y.; Zheng, J. M.; Xu, H.; Wei, G.; Hao, J. S.; Cui, F. D. Bioadhesive polysaccharide in protein delivery system: chitosan nanoparticles improve the intestinal absorption of insulin in vivo. Int. J. Pharm. 2002, 249, 139-147.

(22) Sakiyama, T.; Takata, H.; Kikuchi, M.; Nakanishi, K. Polyelectrolyte complex gel with high $\mathrm{pH}$-sensitivity prepared from dextran sulfate and chitosan. J. Appl. Polym. Sci. 1999, 73, 2227-2233.

(23) Sakiyama, T.; Takata, H.; Toga, T.; Nakanishi, K., pH-sensitive shrinking of a dextran sulfate/chitosan complex gel and its promotion effect on the release of polymeric substances. J. Appl. Polym. Sci. 2001, 81, 667-674.

(24) Serizawa, T.; Yamaguchi, M.; Kishida, A.; Akashi, M. Alternating gene expression in fibroblasts adhering to multilayers of chitosan and dextran sulfate. J. Biomed. Mater Res. 2003, 67, 1060-1063.

(25) Chen, Y.; Mohanraj, V. J.; Parkin, J. E., Chitosan-dextran sulfate nanoparticles for delivery of an anti-angiogenesis peptide. Lett. Pept. Sci. 2004, 10, 621-629.

(26) Sarmento, B.; Ribeiro, A.; Veiga, F.; Ferreira, D. Development and characterization of new insulin containing polysaccharide nanoparticles. Colloid Surf. B 2006, 53, 193-202.

(27) Buschard, K.; Thon, R., Diabetic Animal Models. In Handbook of Laboratory Animal Science; Hau, J., Hoosier, G. L. V., Eds.; CRS Press: Boca Raton, FL, 2003; Vol. II.

(28) Morishita, M.; Goto, T.; Nakamura, K.; Lowman, A. M.; Takayama, K.; Peppas, N. A. Novel oral insulin delivery systems based on complexation polymer hydrogels: Single and multiple administration studies in type 1 and 2 diabetic rats. J. Control. Release 2006, 110 , $587-594$
(29) Bradford, M. M. A rapid and sensitive method for the quantitation of microgram quantities of protein utilizing the principle of proteindye binding. Anal. Biochem. 1976, 72, 248- 254.

(30) Sarmento, B.; Ribeiro, A.; Veiga, F.; Sampaio, P.; Neufeld, R.; Ferreira, D. Alginate-chitosan nanoparticles are effective for oral insulin delivery. Pharm. Res., in press.

(31) Schatz, C.; Lucas, J. M.; Viton, C.; Domard, A.; Pichot, C.; Delair, T. Formation and properties of positively charged colloids based on polyelectrolyte complexes of biopolymers. Langmuir 2004, 20, 7766-7778.

(32) Hartig, S. M.; Carlesso, G.; Davidson, J. M.; Prokop, A. Development of Improved Nanoparticulate Polyelectrolyte Complex Physicochemistry by Nonstoichiometric Mixing of Polyions with Similar Molecular Weights. Biomacromolecules 2007, 8, 265-272.

(33) Ma, Z.; Yeoh, H. H.; Lim, L. Y. Formulation pH modulates the interaction of insulin with chitosan nanoparticles. J. Pharm. Sci. 2002 91, 1396-404.

(34) Sarmento, B.; Ferreira, D. C.; Jorgensen, L.; van de Weert, M. Probing insulin's secondary structure after entrapment into alginate/chitosan nanoparticles. Eur. J. Pharm. Biopharm. 2007, 65, 10-17.

(35) Bendayan, M.; Ziv, E.; Gingras, D.; Ben-Sasson, R.; Bar-On, H.; Kidron, M. Biochemical, and morpho-cytochemical evidence for the intestinal absorption of insulin in control and diabetic rats. Comparison between the effectiveness of duodenal and colon mucosa. Diabetologia 1994, 37, 119-126.

(36) Ziv, E.; Bendayan, M. Intestinal absorption of peptides through the enterocytes. Microsc. Res. Technol. 2000, 49, 346-352.

(37) Morishita, M.; Morishita, I.; Takayama, K.; Machida, Y.; Nagai, T. Site-dependent effect of aprotinin, sodium caprate, Na2EDTA and sodium glycocholate on intestinal absorption of insulin. Biol. Pharm. Bull. 1993, 16, 68-72.

(38) Chen, H.; Langer, R. Oral particulate delivery: status and future trends. Adv. Drug. Deliv. Rev. 1998, 34, 339-350.

(39) Shimoda, J.; Onishi, H.; Machida, Y. Bioadhesive Characteristics of Chitosan Microspheres to the Mucosa of Rat Small Intestine. Drug Dev. Ind. Pharm. 2001, 27, 567-576.

(40) Sakkinen, M.; Marvola, J.; Kanerva, H.; Lindevall, K.; Ahonen, A.; Marvola, M. Are chitosan formulations mucoadhesive in the human small intestine?: An evaluation based on gamma scintigraphy. Int J. Pharm. 2006, 307, 285-291.

(41) Prego, C.; Torres, D.; Alonso, M. J. The potential of chitosan for the oral administration of peptides. Expert Opin. Drug. Deliv. 2005, $2,843-854$

(42) Schnabel, C. A.; Fineberg, S. E.; Kim, D. D. Immunogenicity of xenopeptide hormone therapies. Peptides 2006, 27, 1902-1910.

(43) Florence, A. T. Nanoparticle uptake by the oral route: Fulfilling its potential? Drug Discovery Today 2005, 2, 75-81.

(44) Desai, M. P.; Labhasetwar, V.; Amidon, G.; Levy, R. Gastrointestinal Uptake of Biodegradable Microparticles: Effect of Particle Size. Pharm. Res. 1996, 13, 1838-1845.

(45) Win, K. Y.; Feng, S. S. Effects of particle size and surface coating on cellular uptake of polymeric nanoparticles for oral delivery of anticancer drugs. Biomaterials 2005, 26, 2713-2722.

(46) Janes, K. A.; Calvo, P.; Alonso, M. J. Polysaccharide colloidal particles as delivery systems for macromolecules. Adv. Drug. Deliv. Rev. 2001, 47, 83-97.

(47) Illum, L. Chitosan and Its Use as a Pharmaceutical Excipient. Pharm Res. 1998, 15, 1326-1331.

(48) Buda, A.; Sands, C.; Jepson, M. A. Use of fluorescence imaging to investigate the structure and function of intestinal M cells. Adv. Drug Deliv. Rev. 2005, 57, 123-134.

(49) Cui, F.; Shi, K.; Zhang, L.; Tao, A.; Kawashima, Y. Biodegradable nanoparticles loaded with insulin-phospholipid complex for ora delivery: Preparation, in vitro characterization and in vivo evaluation. J. Control Release 2006, 114, 242-250.

(50) Jung, T.; Kamm, W.; Breitenbach, A.; Kaiserling, E.; Xiao, J. X.; Kissel, T. Biodegradable nanoparticles for oral delivery of peptides: is there a role for polymers to affect mucosal uptake? Eur. J. Pharm. Biopharm. 2000, 50, 147-160.

BM0703923 